\title{
On Uniqueness of Commutative Rings of Weyl Group Invariant Differential Operators
}

By

\author{
Kenji Taniguchi*
}

\begin{abstract}
The uniqueness of commutative rings of classical Weyl group invariant differential operators is discussed. We show this uniqueness for trigonometric or elliptic potential cases under some order conditions. For rational potential cases, counter examples are constructed.
\end{abstract}

\section{§o. Introduction}

It is important and interesting to construct and classify commutative rings of differential operators which contain the Laplacian

$$
H=\frac{1}{2} \sum_{i=1}^{n} \frac{\partial^{2}}{\partial x_{i}^{2}}+R(x) \text {. }
$$

Such rings have been studied from physical points of view. The Toda lattice and the many body problem are examples of such rings ([OP1], [OP2]). On the other hand, to generalize the ring of invariant differential operators on a Riemannian symmetric space, Ochiai, Oshima and Sekiguchi formulated commutative rings of Weyl group $W$ invariant differential operators. For classical Weyl groups, they classified the potential function $R(x)$ and constructed all the higher order operators explicitly ([OS], [OOS $]$ ). Let $\Sigma$ be the reduced root system corresponding to $W$ and $\Sigma^{+}$be a positive system of $\Sigma$. According to them, $R(x)$ can be expressed as

$$
R(x)=\sum_{\alpha \in \Sigma^{+}} u_{\alpha}(\langle\alpha, x\rangle)\left(u_{\alpha}(t)=u_{w \alpha}(t) \text { for any } \alpha \in \Sigma, w \in W\right) .
$$

Moreover, $u_{\alpha}(t)$ 's are some rational, trigonometric or elliptic functions.

Let $H$ be a Laplacian whose potential function is one of those which

Communicated by M. Kashiwara, August 19, 1996.

1991 Mathematics Subject Classification(s) : 58F07

*JSPS Research Fellow

Graduate School of Mathematical Sciences, University of Tokyo, 3-8-1, Komaba, Meguro-ku, Tokyo 153, Japan. 
Ochiai, Oshima and Sekiguchi obtained. In this note, we shall investigate what kind of differential operator $P$ commutes with $H$. Note that we do not assume $P$ to be $W$-invariant nor to have constant symbol (see $\$ 2$ (C3)). The arguments and conclusions differ with the potential functions. In $\$ 4$, we treat the periodic (i.e. trigonometric or elliptic) potential cases and in $\$ 5$ the rational potential cases.

The main result for the periodic cases is the following (Theorem 4.4) :

Theorem 0.1. Let $(W, \Sigma)$ be a pair of a classical Weyl group and the corresponding root system. Let $H=1 / 2 \sum_{i=1}^{n} \partial_{x_{i}}^{2}+\sum_{\alpha \in \Sigma}+u_{\alpha}(\langle\alpha, x\rangle)\left(u_{\alpha}(t)=\right.$ $\left.u_{w \alpha}(t)\right)$ be a Laplacian, where all $u_{\alpha}(t)$ 's are non-constant periodic functions in the classification of Theorem 2.1. Suppose that $P$ is a holomorphic differential operator which is defined on a connected open subset of the domain where $H$ is defined, and commutes with $H$. Assume that the order of $P$ is at most $n$ (resp. $2 n$ ) for the $A_{n-1}$ or $D_{n}$ (resp. $B_{n}$ ) cases. Then $P$ has analytic continuation to the whole domain where $H$ is defined and is contained in the commutative ring constructed by Ochiai, Oshima and Sekiguchi.

Since $P$ is not assumed to be $W$-invariant, this is a stronger version of the uniqueness theorem of such commutative rings ([OS, Theorem 3.2]). Moreover, as a corollary of this theorem, if $P_{1}$ and $P_{2}$ are differential operators satisfying the above conditions and $\left[H, P_{1}\right]=\left[H, P_{2}\right]=0$, then $\left[P_{1}, P_{2}\right]=0$.

The author expects that, under weaker order assumptions, any differential operator $P$ which commutes with $H$ is contained in the commutative ring of Ochiai-Oshima-Sekiguchi. For details, see Remark 4.3.

In the rational potential cases, the situation is a little different from these periodic cases. As an example, we shall construct all the differential operators which commute with $H=1 / 2 \sum_{i=1}^{n} \partial^{2} / \partial_{x_{i}}^{2}+\sum_{1 \leq i<j \leq n}\left\{C_{1}\left(x_{i}-x_{j}\right)^{-2}+C_{2}\right\} \quad\left(A_{n-1^{-}}\right.$ type Laplacian) and of order at most 2. Moreover, using these operators, we prove that, if $n>3$, then there is no commutative ring satisfying (C1)-(C5) in $\$ 2$ other than those which Oshima and Sekiguchi constructed in [OS]. The result is as follows (Proposition 5.1 and Theorem 5.3):

Theorem 0.2. Suppose that $W=\mathfrak{S}_{n}$ and $u(t)=C_{1} t^{-2}+C_{2}, C_{1} \neq 0$.

(1) Let $P$ be a holomorphic differential operator of order at most 2 which is defined on $\left\{x \in C^{n} ;|x|<r\right\} \cap\left(C^{n}-\bigcup_{1 \leq i<j \leq n}\left\{x \in C^{n} ; x_{i}=x_{j}\right\}\right)$ for some $r \in \boldsymbol{R}_{>0}$, and commutes with $H$. Then $P$ is $\subseteq_{n}$-invariant, has analytic continuation to $C^{n}-\cup_{1 \leq i<j \leq n}\left\{x \in C^{n} ; x_{i}=x_{j}\right\}$, and is a linear combination of $1, \Delta_{1}=\sum_{i=1}^{n} \partial_{x_{i}}, \Delta_{1}^{2}, H, P_{1}, P_{2}$ and $P_{3}$, where 


$$
\begin{aligned}
P_{1}:= & \sum_{1 \leq i<j \leq n}\left(x_{j} \partial_{x_{i}}-x_{i} \partial_{x_{j}}\right)^{2}+2 C_{1} \sum_{\substack{1 \leq i<j \leq n \\
1 \leq p \leq n}} x_{p}^{2}\left(x_{i}-x_{j}\right)^{-2}, \\
P_{2}:= & \sum_{1 \leq i \neq j \neq k \neq i \leq n}\left(x_{j} \partial_{x_{i}}-x_{i} \partial_{x_{j}}\right)\left(x_{k} \partial_{x_{i}}-x_{i} \partial_{x_{k}}\right) \\
& +2 C_{1} \sum_{1 \leq i<j \leq n}\left(2 \sum_{1 \leq p<q \leq n} x_{p} x_{q}-\sum_{p=1}^{n} x_{p}^{2}\right)\left(x_{i}-x_{j}\right)^{-2}, \\
P_{3}:= & \sum_{1 \leq i<j \leq n}\left(x_{j} \partial_{x_{i}}-x_{i} \partial_{x_{j}}\right)\left(\partial_{x_{i}}-\partial_{x_{j}}\right)+2 C_{1} \sum_{\substack{1 \leq i<j \leq n \\
1 \leq p \leq n}} x_{p}\left(x_{i}-x_{j}\right)^{-2} .
\end{aligned}
$$

(2) If $n>3$, then there exists no ring satisfying (C1)-(C5) in \$2 other than what Oshima and Sekiguchi constructed in [OS].

(3) If $n=3$, then $\Delta_{3}=\partial_{x_{1}} \partial_{x_{2}} \partial_{x_{3}}-C_{1} \sum_{\substack{1 \leq i \leq 3 \\ 1 \leq j<k \leq 3, j, k \neq i}}\left(x_{j}-x_{k}\right)^{-2} \partial_{x_{i}}, \Delta_{1}$ and $H$ generate a commutative ring. This is proved in [OS]. Define

$$
\begin{array}{r}
P=\left\{\left(x_{2}-x_{3}\right) \partial_{x_{1}}+\left(x_{3}-x_{1}\right) \partial_{x_{2}}+\left(x_{1}-x_{2}\right) \partial_{x_{3}}\right\}^{2} \\
+2 C_{1} \sum_{\substack{1 \leq p<q \leq 3 \\
1 \leq i<j \leq 3}}\left(x_{p}-x_{q}\right)^{2}\left(x_{i}-x_{j}\right)^{-2} .
\end{array}
$$

Then, $\Delta_{1}, H$ and $\Delta_{3}+\lambda P$ also generate a commutative ring satisfying (C1)-(C5) in $\$ 2$ for any $\lambda \in C$.

For the corresponding results for $B_{n}$ and $D_{n}$ cases, see Remark 5.4.

\section{Acknowledgements}

The author would like to express his gratitude to T. Oshima and H. Ochiai for discussion, kind explanations on their papers and comments.

\section{§1. General Notation}

We fix an orthonormal basis $\left\{e_{1}, \cdots, e_{n}\right\}$ of $\boldsymbol{R}^{n}$. Let $W$ be a Weyl group and $\sum$ be the corresponding reduced root system. Root systems $\Sigma$ of type $A_{n-1}$, $B_{n}$ and $D_{n}$ are realized in $\boldsymbol{R}^{n}$ and we choose positive systems of them by ;

$$
\begin{aligned}
& A_{n-1} \text {-type : } \Sigma^{+}=\left\{e_{i}-e_{j} ; 1 \leq i<j \leq n\right\} \text {, }
\end{aligned}
$$

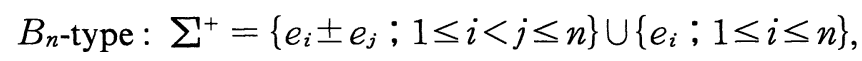

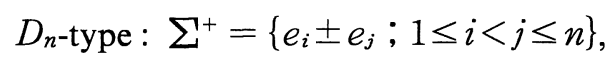


and fix them.

In this paper, we treat holomorphic differential operators on $\boldsymbol{C}^{n}=\boldsymbol{R}^{n} \otimes_{R} C$ and use the coordinate system $\left(x_{1}, \cdots, x_{n}\right)$ with $\sum_{i=1}^{n} x_{i} e_{i} \in \mathbb{C}^{n}$.

We put $\partial_{x_{i}}=\partial / \partial x_{i}(1 \leq i \leq n)$. For $\alpha \in \sum$ and $x=\left(x_{1}, \cdots, x_{n}\right) \in \mathbb{C}^{n}$, let $\langle\alpha$, $x\rangle$ be the coupling. For example, if $\alpha=e_{i}-e_{j}$, then $\langle\alpha, x\rangle=x_{i}-x_{j}$. The norm $|\alpha|$ of a root $\alpha$ is defined by $|\alpha|=\sqrt{\langle\alpha, \alpha\rangle}$. Similarly, let $\left\langle\alpha, \partial_{x}\right\rangle$ be the coupling of $\alpha$ and $\partial_{x}=:\left(\partial_{x_{1}}, \cdots, \partial_{x_{n}}\right)$.

For a multi-index $p=\left(p_{1}, \cdots, p_{n}\right) \in \boldsymbol{Z}_{\geq 0}^{n}$, we put $\partial_{x}^{p_{0}}=\partial_{x_{1}}^{p_{1}} \cdots \partial_{x_{n}}^{p_{n}}$ and $|p|:=$ $\sum_{i=1}^{n} p_{i}$

Let $P$ be a differential operator on some open subset of $C^{n}$. We decompose $P$ into $P=\sum_{k=0}^{m} P_{k}$, where $P_{k}=\sum_{|p|=k} a_{p}(x) \partial_{x}^{p}$. We define $\widetilde{P}_{k}:=\sum_{|p|=k} a_{p}(x) \xi^{p}$ $\left(\xi=\left(\xi_{1}, \cdots, \xi_{n}\right)\right)$, and call it the $k$-th symbol of $P$. Especially, we call $\widetilde{P}_{m}$ the principal symbol of $P$. For $\xi$, we define $\partial_{\xi i}, \partial_{\xi}^{p},\langle\alpha, \xi\rangle$ and $\left\langle\alpha, \partial_{\xi}\right\rangle$ analogously to those of $x$.

For differentiable $2 n$-variable functions $f(x, \xi)$ and $g(x, \xi)$, the Poisson bracket $\{, \quad\}$ is defined by

$$
\{f, g\}=\sum_{i=1}^{n}\left(\left(\partial_{\xi_{i}} f\right)\left(\partial_{x_{i}} g\right)-\left(\partial_{x_{i}} f\right)\left(\partial_{\xi_{i}} g\right)\right) .
$$

Remark 1.1. If two differential operators $P$ and $Q$ commute each other, then the Poisson bracket of their principal symbols $\sigma(P)$ and $\sigma(Q)$ is zero.

\section{§2. Commutative Rings of Weyl Group Invariant Differential Operators}

In this section, we review the results in [OS] and [OOS].

Let $C$ be a commutative ring of differential operators satisfying the following conditions :

(C1) Elements of $C$ are holomorphic differential operators on some appropriate $W$-invariant open connected subset $\Omega$ of $C^{n}$ with $0 \in \bar{\Omega}$.

(C2) Elements of $C$ commute mutually.

(C3) Elements of $C$ are $W$-invariant and the principal symbols of them are constant with respect to coordinates $\left\{x_{i}\right\}$.

(C4) $C$ contains a Laplacian $H=1 / 2 \sum_{i=1}^{n} \partial_{x_{i}}^{2}+R(x)$.

(C5) Principal symbols of elements of $C$ generate $C[\xi]^{W}$.

When $W$ is a classical Weyl group, Ochiai, Oshima and Sekiguchi determined the potential function $R(x)$ and constructed higher order generators of $C$ in $[\mathrm{OS}]$ and $[\mathrm{OOS}]$.

Theorem 2.1 ([OOS, Theorem 1]). $R(x)$ can be expressed as

$$
R(x)=\sum_{1 \leq i<j \leq n} u\left(x_{i}-x_{j}\right), \quad \quad \text { if } W \text { is of type } A_{n-1},
$$




$$
\begin{array}{ll}
R(x)=\sum_{1 \leq i<j \leq n}\left(u\left(x_{i}-x_{j}\right)+u\left(x_{i}+x_{j}\right)\right)+\sum_{i=1}^{n} v\left(x_{i}\right), & \text { if } W \text { is of type } B_{n}, \\
R(x)=\sum_{1 \leq i<j \leq n}\left(u\left(x_{i}-x_{j}\right)+u\left(x_{i}+x_{j}\right)\right), & \text { if } W \text { is of type } D_{n} .
\end{array}
$$

The above functions $u(t)$ and $v(t)$ are as follows:

If $W$ is of type $A_{n-1}$ with $n \geq 3$,

$$
u(t)=C_{1} \wp(t)+C_{2} .
$$

If $W$ is of type $B_{n}$ with $n \geq 3$,

$$
\left\{\begin{array}{l}
u(t)=C_{1} \wp(t)+C_{2} \\
v(t)=\frac{C_{3} \wp(t)^{4}+C_{4} \wp(t)^{3}+C_{5} \wp(t)^{2}+C_{6} \wp(t)+C_{7}}{\wp^{\prime}(t)^{2}}
\end{array}\right.
$$

or

$$
u(t)=C_{1} t^{-2}+C_{2} t^{2}+C_{3} \text { and } v(t)=C_{4} t^{-2}+C_{5} t^{2}+C_{6}
$$

or

$$
u(t)=C_{1} \text { and } v(t) \text { is any even function. }
$$

If $W$ is of type $D_{n}$ with $n \geq 4$, then $u$ is (2.2) or (2.3).

If $W$ is of type $B_{2}$ then $(u(t), v(t))$ is (2.2) or (2.3) or (2.4) or

$$
\left\{\begin{array}{l}
u(t)=\frac{C_{3} \wp(t / 2)^{4}+C_{4} \wp(t / 2)^{3}+C_{5} \wp(t / 2)^{2}+C_{6} \wp(t / 2)+C_{7}}{\gamma^{\prime}(t / 2)^{2}} \\
v(t)=C_{1} \wp(t)+C_{2}
\end{array}\right.
$$

or

$$
\left\{\begin{array}{l}
u(t)=C_{1} \wp(t)+C_{2} \frac{\left(\wp(t / 2)-e_{3}\right)^{2}}{\wp^{\prime}(t / 2)^{2}}+C_{3}, \\
v(t)=C_{4} \wp(t)+\frac{C_{5}}{\wp(t)-e_{3}}+C_{6}
\end{array}\right.
$$

or

$$
v(t)=C_{1} \text { and } u(t) \text { is any even function. }
$$

Here, $C_{i}$ 's are arbitrary complex numbers and $\wp(t)$ is the Weierstrass' elliptic function $\gamma\left(t \mid 2 \omega_{1}, 2 \omega_{2}\right)$ with primitive half-periods $\omega_{1}$ and $\omega_{2}$ which allowed to be infinity, and $e_{3}$ is a complex number satisfying $\gamma^{\prime 2}=4\left(\wp-e_{1}\right)\left(\wp-e_{2}\right)\left(\wp-e_{3}\right)$ (see $[\mathrm{WW}]$ ).

Note that $\wp(t \mid \infty, \infty)=t^{-2}$, and $\wp(t \mid \sqrt{-1} \pi, \infty)=\sinh ^{-2} t+1 / 3$. 


\section{§3. Basic Results}

In this note, as we mentioned in the introduction, we investigate what kind of differential operator $P$ commutes with $H=1 / 2 \sum_{i=1}^{n} \partial_{x_{i}}^{2}+\sum_{\alpha \in \Sigma+} u_{\alpha}(\langle\alpha, x\rangle)$. Here, (1) $u_{\alpha}(t)=u_{w \alpha}(t)$ for $\alpha \in \Sigma$ and $w \in W$, (2) $u_{\alpha}(t)$ 's are given by the classification in Theorem 2.1.

Suppose that $P$ is an $m$-th order holomorphic differential operator which commutes with $H$, and is defined on a connected open subset of the domain where $H$ is defined. Then, by Remark 1.1,

$$
\left\{\frac{1}{2} \sum_{i=1}^{n} \xi_{i}^{2}, \widetilde{P}_{m}\right\}=\sum_{i=1}^{n} \xi_{i} \partial_{x_{i}} \widetilde{P}_{m}=0
$$

For notational convenience, put $\mathscr{D}:=\sum_{i=1}^{n} \xi_{i} \partial_{x_{i}}$ and $\mathscr{L}:=\sum_{i=1}^{n} \partial_{x_{i}} \partial_{\xi_{i}}$. We define $y_{1}, \cdots, y_{n}$ by

$$
y_{j}=\frac{x_{j}}{\xi_{j}}-\frac{x_{j+1}}{\xi_{j+1}}(1 \leq j \leq n-1) .
$$

The following lemma is elementary.

Lemma 3.1. By the above notations, we have the following formulae:

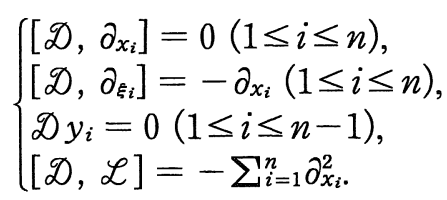

For $\alpha \in \Sigma^{+}$and a holomorphic function $f(t)$,

$$
f^{\prime}(\langle\alpha, x\rangle)=\mathscr{D}\left(\frac{f(\langle\alpha, x\rangle)}{\langle\alpha, \xi\rangle}\right)
$$

\section{Corollary 3.2.}

(1) If $\widetilde{Q}(x, \xi)$ satisfies $\left\{1 / 2 \sum_{i=1}^{n} \xi_{i}^{2}, \widetilde{Q}\right\}=0$, then $\widetilde{Q}$ is a function of $\xi$ and $y_{1}, \cdots, y_{n-1}$.

(2) Suppose that a holomorphic differential operator $Q$ commutes with $H$, and is defined on a connected open subset of the domain where $H$ is defined. Then the principal symbol $\sigma(Q)$ of $Q$ is an element of $\mathscr{R}:=$ $C\left[\xi_{i}(1 \leq i \leq n), x_{i} \xi_{j}-x_{j} \xi_{i}(1 \leq i<j \leq n)\right]$, and the highest order term of $Q$ has analytic continuation to the whole space $C^{n}$.

(3) If $\widetilde{Q}(x, \xi) \in \mathscr{R}$ is symmetric with respect to $\xi$, i.e. $\widetilde{Q}(x, \sigma(\xi))=\widetilde{Q}(x, \xi)$ for any $\sigma \in \Im_{n}$, then $\widetilde{Q} \in C[\xi]$.

Proof. (1) Since $\left\{1 / 2 \sum_{i=1}^{n} \xi_{i}^{2}, \quad \widetilde{Q}\right\}=\sum_{i=1}^{n} \xi_{i} \partial_{x_{i}} \widetilde{Q}=\mathscr{D} \widetilde{Q}=0$, (1) follows 
from (3.3).

(2) By Remark 1.1, $D \sigma(Q)=0$. Moreover, from (3.3), $\partial_{x_{i}} \sigma(Q)=-[D$, $\left.\partial_{\xi_{i}}\right] \sigma(Q)=-D \partial_{\xi_{i}} \sigma(Q)$. By induction, we can prove $\partial_{x}^{p} \sigma(Q)=$ $(-1)^{|p|} D^{|p|} \partial_{\xi}^{p} \sigma(Q) /|p|$ !. Since $\sigma(Q)$ is a polynomial with respect to $\xi, \sigma(Q)$ is also a polynomial with respect to $x$. Then (2) follows from (1).

(3) By the definition of $\mathscr{R}, \widetilde{Q}(\xi, x)$ satisfies $D \widetilde{Q}(\xi, x)=0$. Since $\widetilde{Q}$ is symmetric with respect to $\xi, \xi_{j} \partial_{x_{i}} \widetilde{Q}(\xi, x)+\xi_{i} \partial_{x_{j}} \widetilde{Q}(\xi, x)+\sum_{\substack{p=1 \\ p \neq i, j}}^{n} \xi_{p} \partial_{x_{p}} \widetilde{Q}(\xi, x)=0$.

By these two equalities, we have $\left(\left(\partial_{x_{i}}-\partial_{x_{j}}\right) \widetilde{Q}\right)(\xi, x)=0$ for any $1 \leq i, j \leq n$. This means $\widetilde{Q}=\widetilde{Q}\left(\xi, \sum_{i=1}^{n} x_{i}\right)$ and $D \widetilde{Q}=\left(\sum_{i=1}^{n} \xi_{i}\right) \widetilde{Q}^{\prime}\left(\xi, \sum_{i=1}^{n} x_{i}\right)=0$, where $\widetilde{Q}^{\prime}(\xi, t)$ $=d \widetilde{Q}(\xi, t) / d t$. Then $\widetilde{Q}$ is constant with respect to $x$.

(3.1) is equivalent to

$$
D \widetilde{P}_{m}=0 \text {. }
$$

The $m$-th order term of $[H, P]=0$ is equivalent to

$$
\frac{1}{2} \sum_{i=1}^{n} \partial_{x_{i}}^{2} \widetilde{P}_{m}+\left\{\frac{1}{2} \sum_{i=1}^{n} \xi_{i}^{2}, \widetilde{P}_{m-1}\right\}=0
$$

then $D \widetilde{P}_{m-1}=-1 / 2 \sum_{i=1}^{n} \partial_{x_{i}}^{2} \widetilde{P}_{m}$. From (3.3) and (3.5),

$$
\mathscr{D}\left(\frac{1}{2} \mathscr{L} \widetilde{P}_{m}\right)=\frac{1}{2}[\mathscr{D}, \mathscr{L}] \widetilde{P}_{m}+\frac{1}{2} \mathscr{L} \mathscr{D} \widetilde{P}_{m}=-\frac{1}{2} \sum_{i=1}^{n} \partial_{x_{i}}^{2} \widetilde{P}_{m}
$$

Then $\widetilde{P}_{m-1}$ can be expressed as :

$$
\widetilde{P}_{m-1}=\frac{1}{2} \mathscr{L} \widetilde{P}_{m}+\widetilde{Q}_{m-1}
$$

with $\widetilde{Q}_{m-1} \in \mathscr{R}\left(=C\left[\xi, x_{j} \xi_{i}-x_{i} \xi_{j}\right]\right)$.

Furthermore, we shall investigate the $(m-1)$-st order term of $[H, P]=0$.

This term implies

$$
\frac{1}{2} \sum_{i=1}^{n} \partial_{x_{i}}^{2} \widetilde{P}_{m-1}+\left\{\frac{1}{2} \sum_{i=1}^{n} \xi_{i}^{2}, \widetilde{P}_{m-2}\right\}=\left\{\widetilde{P}_{m}, R(x)\right\} .
$$

Since

$$
\begin{aligned}
&\left\{\widetilde{P}_{m}, R(x)\right\}= \sum_{i=1}^{n} \partial_{\xi_{i}} \widetilde{P}_{m} \cdot \partial_{x_{i}}\left(\sum_{\alpha \in \Sigma^{+}} u_{\alpha}(\langle\alpha, x\rangle)\right) \\
&= \sum_{\alpha \in \Sigma^{+}}\left\langle\alpha, \partial_{\xi}\right\rangle \widetilde{P}_{m} u_{\alpha}^{\prime}(\langle\alpha, x\rangle) \\
& \mathscr{D} \widetilde{P}_{m-2}=-\frac{1}{4} \sum_{i=1}^{n} \partial_{x_{i}}^{2} \mathcal{L} \widetilde{P}_{m}-\frac{1}{2} \sum_{i=1}^{n} \partial_{x_{i}}^{2} \widetilde{Q}_{m-1} \\
& \quad+\sum_{\alpha \in \Sigma^{+}}\left\langle\alpha, \partial_{\xi}\right\rangle \widetilde{P}_{m} u_{\alpha}^{\prime}(\langle\alpha, x\rangle)
\end{aligned}
$$

By (3.3), 


$$
\mathscr{D}\left(\frac{1}{8} \mathscr{L}^{2} \widetilde{P}_{m}+\frac{1}{2} \mathscr{L} \widetilde{Q}_{m-1}\right)=-\frac{1}{4} \sum_{i=1}^{n} \partial_{x_{i}}^{2} \mathscr{L} \widetilde{P}_{m}-\frac{1}{2} \sum_{i=1}^{n} \partial_{x_{i}}^{2} \widetilde{Q}_{m-1}
$$

We integrate the third term in the right hand side of (3.7). Since $D \widetilde{P}_{m}=$ 0 ,

$$
\mathscr{D}\left(\left\langle\alpha, \partial_{\xi}\right\rangle \widetilde{P}_{m}\right)=\left[D,\left\langle\alpha, \partial_{\xi}\right\rangle\right] \widetilde{P}_{m}=-\left\langle\alpha, \partial_{x}\right\rangle \widetilde{P}_{m}
$$

By (3.4), we have

$$
\begin{aligned}
& \mathscr{D}\left(\frac{\left\langle\alpha, \partial_{\xi}\right\rangle \widetilde{P}_{m}}{\langle\alpha, \xi\rangle} u_{\alpha}(\langle\alpha, x\rangle)+\frac{\left\langle\alpha, \partial_{x}\right\rangle \widetilde{P}_{m}}{\langle\alpha, \xi\rangle^{2}} U_{\alpha}(\langle\alpha, x\rangle)\right) \\
& \quad=\left\langle\alpha, \partial_{\xi}\right\rangle \widetilde{P}_{m} u_{\alpha}^{\prime}(\langle\alpha, x\rangle) .
\end{aligned}
$$

Here, $U_{\alpha}(t)$ is a primitive function of $u_{\alpha}(t)$. Then, we have proved the following proposition.

Proposition 3.3. $\quad \widetilde{P}_{m-2}$ can be expressed as :

$$
\begin{aligned}
\widetilde{P}_{m-2}= & \frac{1}{8} \mathscr{L}^{2} \widetilde{P}_{m}+\frac{1}{2} \mathscr{L} \widetilde{Q}_{m-1}+\widetilde{Q}_{m-2} \\
& +\sum_{\alpha \in \Sigma^{+}}\left\{\frac{\left\langle\alpha, \partial_{\xi}\right\rangle \widetilde{P}_{m}}{\langle\alpha, \xi\rangle} u_{\alpha}(\langle\alpha, x\rangle)+\frac{\left\langle\alpha, \partial_{x}\right\rangle \widetilde{P}_{m}}{\langle\alpha, \xi\rangle^{2}} U_{\alpha}(\langle\alpha, x\rangle)\right\} .
\end{aligned}
$$

Here, since $\widetilde{P}_{m-2}, \mathscr{L}^{2} \widetilde{P}_{m}$ and $\mathscr{L} \widetilde{Q}_{m-1}$ are polynomials with respect to $\xi$ and $\mathscr{D} \widetilde{Q}_{m-2}=0, \widetilde{Q}_{m-2} \in \Sigma_{\alpha \in \Sigma^{+}} \frac{1}{\langle\alpha, \xi\rangle^{2}} \mathscr{R}$.

\section{§4. Uniqueness of Commutative Rings with Periodic Potentials}

In this section we assume that

$$
\begin{array}{r}
\text { for any } \alpha \in \Sigma^{+}, u_{\alpha}(t) \text { is a non-trivial periodic function } \\
\text { in the classification in Theorem } 2.1 .
\end{array}
$$

Proposition 4.1. Under the assumption (4.1), $\left\langle\alpha, \partial_{\xi}\right\rangle \widetilde{P}_{m}$ is divisible by $\langle\alpha$, $\xi\rangle$ and $\left\langle\alpha, \partial_{x}\right\rangle \widetilde{P}_{m}$ is divisible by $\langle\alpha, \xi\rangle^{2}$.

Proof. First, notice that the first and second terms of the right hand side of (3.8) and $\widetilde{P}_{m-2}$ are polynomials with respect to $\xi$. Then there exists a polynomial $\widetilde{Q}_{m-2}^{\prime} \in \mathscr{R}$ such that $\left\langle\alpha, \partial_{x}\right\rangle \widetilde{P}_{m} U_{\alpha}(\langle\alpha, x\rangle)+\widetilde{Q}_{m-2}^{\prime}$ is divisible by $\langle\alpha$, $\xi\rangle$, i.e.

$$
\lim _{\langle\alpha, \xi\rangle \rightarrow 0}\left\{\left\langle\alpha, \partial_{x}\right\rangle \widetilde{P}_{m} U_{\alpha}(\langle\alpha, x\rangle)+\widetilde{Q}_{m-2}^{\prime}\right\}=0
$$


Suppose that $\lim _{\langle\alpha, \xi\rangle \rightarrow 0}\left\langle\alpha, \partial_{x}\right\rangle \widetilde{P}_{m} U_{\alpha}(\langle\alpha, x\rangle) \neq 0$. If we pay attention to a coefficient of an appropriate monomial with respect to $\xi$ in (4.2), there exist polynomials $f(x) \neq 0$ and $g(x)$ of $x$ such that

$$
f(x) U_{\alpha}(\langle\alpha, x\rangle)+g(x)=0 .
$$

It follows that $U_{\alpha}(t)$ is a rational function and this contradicts the assumption (4.1). Then $\left\langle\alpha, \partial_{x}\right\rangle \widetilde{P}_{m}$ is divisible by $\langle\alpha, \xi\rangle$.

Next, there exists a polynomial $\widetilde{Q}_{m-2}^{\prime \prime} \in \mathscr{R}$ such that

$$
\lim _{\langle\alpha, \xi\rangle \rightarrow 0}\left\{\left\langle\alpha, \partial_{\xi}\right\rangle \widetilde{P}_{m} u_{\alpha}(\langle\alpha, x\rangle)+\frac{\left\langle\alpha, \partial_{x}\right\rangle \widetilde{P}_{m}}{\langle\alpha, \xi\rangle} U_{\alpha}(\langle\alpha, x\rangle)+\widetilde{Q}_{m-2}^{\prime \prime}\right\}=0 .
$$

Let $x_{1}^{\prime}:=\langle\alpha, x\rangle$ and $x^{\prime}:=\left(x_{2}^{\prime}, \cdots, x_{n}^{\prime}\right)$ be new variables which satisfy $C[\langle\alpha, x\rangle$, $\left.x^{\prime}\right]=C[x]$ and $\left\langle\alpha, \partial_{x}\right\rangle x_{j}^{\prime}=0$ for $2 \leq j \leq n$. For example, if $\alpha=e_{1}-e_{2}$, then $x_{1}^{\prime}=$ $x_{1}-x_{2}, x_{2}^{\prime}=x_{1}+x_{2}$ and $x_{j}^{\prime}=x_{j}(3 \leq j \leq n)$. We define $\xi_{1}^{\prime}:=\langle\alpha, \xi\rangle, \xi^{\prime}:=\left(\xi_{2}^{\prime}, \cdots\right.$, $\left.\xi_{n}^{\prime}\right)$ analogously.

Since $\left\langle\alpha, \partial_{x}\right\rangle \widetilde{P}_{m}$ is divisible by $\langle\alpha, \xi\rangle$ and $\widetilde{P}_{m}$ is a polynomial of $x$ and $\xi$, $\widetilde{P}_{m}$ is expressed as

$$
\widetilde{P}_{m}=\langle\alpha, \xi\rangle S_{1}\left(\langle\alpha, \xi\rangle, \xi^{\prime},\langle\alpha, x\rangle, x^{\prime}\right)+S_{2}\left(\xi^{\prime}, x^{\prime}\right),
$$

with some polynomial functions $S_{1}\left(s, \xi^{\prime}, t, x^{\prime}\right), S_{2}\left(\xi^{\prime}, x^{\prime}\right)$. By this expression,

$$
\begin{aligned}
\lim _{\langle\alpha, \xi\rangle \rightarrow 0} \frac{\left\langle\alpha, \partial_{x}\right\rangle \widetilde{P}_{m}}{\langle\alpha, \xi\rangle} & =|\alpha|^{2}\left(\partial_{t} S_{1}\right)\left(0, \xi^{\prime},\langle\alpha, x\rangle, x^{\prime}\right) \\
& =|\alpha|^{-2}\left\langle\alpha, \partial_{x}\right\rangle \lim _{\langle\alpha, \xi\rangle \rightarrow 0}\left\langle\alpha, \partial_{\xi}\right\rangle \widetilde{P}_{m} .
\end{aligned}
$$

Suppose that $\left\langle\alpha, \partial_{\xi}\right\rangle \widetilde{P}_{m}$ is not divisible by $\langle\alpha, \xi\rangle$. Then there are polynomials $f_{\alpha}(x) \neq 0$ and $g_{\alpha}(x)$ of $x$ such that

$$
f_{\alpha}(x) u_{\alpha}(\langle\alpha, x\rangle)+|\alpha|^{-2}\left(\left\langle\alpha, \partial_{x}\right\rangle f_{\alpha}\right)(x) U_{\alpha}(\langle\alpha, x\rangle)=g_{\alpha}(x) .
$$

For a constant $\omega$ and a function $\phi(x)=\phi\left(x_{1}, \cdots, x_{n}\right)$, we denote $\phi\left(x+\omega e_{i}\right)=$ $\phi\left(x_{1}, \cdots, x_{i}+\omega, \cdots, x_{n}\right)$.

Suppose that $\omega$ is a non-zero period of $u_{\alpha}(t)$. Then there exists a constant $C_{\omega}$ such that $U_{\alpha}(t+\omega)=U_{\alpha}(t)+C_{\omega}$. Substituting $x+\omega e_{i}$ for $x$ of (4.5), we have

$$
\begin{gathered}
f_{\alpha}\left(x+\omega e_{i}\right) u_{\alpha}(\langle\alpha, x\rangle)+|\alpha|^{-2}\left(\left\langle\alpha, \partial_{x}\right\rangle f_{\alpha}\right)\left(x+\omega e_{i}\right) U_{\alpha}(\langle\alpha, x\rangle) \\
=g_{\alpha}\left(x+\omega e_{i}\right)-C_{\omega}|\alpha|^{-2}\left(\left\langle\alpha, \partial_{x}\right\rangle f_{\alpha}\right)\left(x+\omega e_{i}\right) .
\end{gathered}
$$

Here we used the fact that $\left\langle\alpha, e_{i}\right\rangle \in \boldsymbol{Z}$ for any $\alpha \in \sum$ since $\sum$ is of $A_{n-1}, B_{n}$ or $D_{n}$-type. By assumption (4.1), $u_{\alpha}$ is not a rational function. Then (4.5) and (4. 6) implies 


$$
\operatorname{det}\left(\begin{array}{cc}
f_{\alpha}(x) & |\alpha|^{-2}\left(\left\langle\alpha, \partial_{x}\right\rangle f_{\alpha}\right)(x) \\
f_{\alpha}\left(x+\omega e_{i}\right) & |\alpha|^{-2}\left(\left\langle\alpha, \partial_{x}\right\rangle f_{\alpha}\right)\left(x+\omega e_{i}\right)
\end{array}\right)=0
$$

Since $f_{\alpha} \neq 0$,

$$
\frac{\left(\left\langle\alpha, \partial_{x}\right\rangle f_{\alpha}\right)(x)}{f_{\alpha}(x)}=\frac{\left(\left\langle\alpha, \partial_{x}\right\rangle f_{\alpha}\right)\left(x+\omega e_{i}\right)}{f_{\alpha}\left(x+\omega e_{i}\right)}
$$

This implies $\left(\left\langle\alpha, \partial_{x}\right\rangle f_{\alpha}\right)(x) / f_{\alpha}(x)$ is a rational periodic function, i.e. a constant function with respect to each $x_{i}(1 \leq i \leq n)$. But since $f_{\alpha}$ is a polynomial function, $f_{\alpha}$ is a constant function. Since $u_{\alpha}$ is not a rational function, (4.5) implies $f_{\alpha} \equiv 0$. This contradicts our assumption, and we have proved that $\langle\alpha$, $\left.\partial_{\xi}\right\rangle \widetilde{P}_{m}$ is divisible by $\langle\alpha, \xi\rangle$. Using (3.8) once more, we conclude that $\langle\alpha$, $\left.\partial_{x}\right\rangle \widetilde{P}_{m}$ is divisible by $\langle\alpha, \xi\rangle^{2}$.

Proposition 4.2. Let $P(x, \xi)=\sum_{|p|=k} C_{p}(x) \xi^{p}$ be a polynomial with respect to $\xi$, and suppose $\lim _{\langle\alpha, \xi\rangle \rightarrow 0}\left\langle\alpha, \partial_{\xi}\right\rangle P(x, \xi)=0$ for any $\alpha \in \Sigma^{+}$.

Assume that $P(x, \xi)$ satisfies at least one of the following conditions:

$$
\begin{aligned}
& \text { The degree of } P(x, \xi) \text { with respect to } \xi \text { is } \\
& \left\{\begin{array}{cc}
\leq n & \left(\left(W, \sum\right) \text { is } A_{n-1} \text { or } D_{n-t y p e}\right) \\
\leq 2 n & \left((W, \Sigma) \text { is } B_{n} \text {-type }\right)
\end{array}\right.
\end{aligned}
$$

(4.8) If all the $p_{i}$ 's of $p=\left(p_{1}, \cdots, p_{n}\right)$ are non-zero, $c_{p}(x)=0$.

Then, $P(x, \xi)$ is $W$-invariant with respect to $\xi$, i.e. $P(x, \sigma(\xi))=P(x, \xi)$ for any $\sigma \in W$.

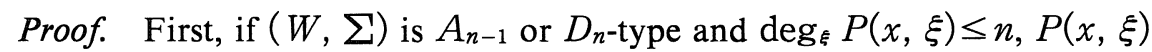
satisfies (4.8) modulo the $W$-invariant term $\xi_{1} \cdots \xi_{n}$. In the case of $B_{n}$-type, $p_{i}$ $=0$ or $p_{i} \geq 2$ for each $i$ since $\lim _{\left\langle e_{i}, \xi\right\rangle \rightarrow 0}\left\langle e_{i}, \partial_{\xi}\right\rangle P(x, \xi)=\lim _{\xi_{i} \rightarrow 0} \partial_{\xi i} P(x, \xi)=0$. It follows that, if $\operatorname{deg}_{\xi} P(x, \xi) \leq 2 n, P(x, \xi)$ satisfies (4.8) modulo the $W$ -

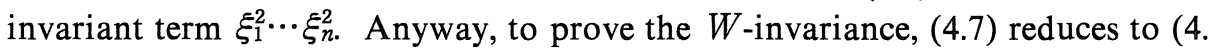
8).

Step 1. $S_{3}$-invariance.

First, we prove this proposition for the case $n=3$.

We denote $P(x, \xi)$ by

$$
P(x, \xi)=\sum_{p_{1}+p_{2}+p_{3}=k} c_{p_{1}, p_{2}, p_{3}}(x) \xi_{1}^{p_{1}} \xi_{2}^{p_{2}} \xi_{3}^{p_{3}} .
$$

To prove $\mathfrak{S}_{3}$-invariance, we apply Proposition 4.1 for $\alpha=e_{i}-e_{j}$. The coefficient of $\xi_{i}^{l-1} \xi_{h}^{h-l}$ in $\lim _{\xi_{j \rightarrow \xi_{i}}}\left(\partial_{\xi_{i}}-\partial_{\xi_{j}}\right) P(x, \xi)=0$ is 


$$
\begin{cases}\sum_{p=0}^{l}(2 p-l) c_{p, l-p, k-l}(x)=0 & (1 \leq l \leq k) \\ \sum_{p=0}^{l}(2 p-l) c_{k-l, p, l-p}(x)=0 & (1 \leq l \leq k) \\ \sum_{p=0}^{l}(2 p-l) c_{p, k-l, l-p}(x)=0 & (1 \leq l \leq k)\end{cases}
$$

We shall prove

$$
\left\{\begin{array}{l}
c_{0, i, k-i}(x)=c_{i, 0, k-i}(x) \\
c_{0, k-i, i}(x)=c_{i, k-i, 0}(x) \\
c_{k-i, 0, i}(x)=c_{k-i, i, 0}(x)
\end{array}\right.
$$

by induction on $i$.

For $i=1,2$, this follows from (4.9) with $l=1,2$. Next, the first equality of (4.9) with $l=i$ is

$$
i\left(c_{i, 0, k-i}(x)-c_{0, i, k-i}(x)\right)+\sum_{p=1}^{i-1}(2 p-i) c_{p, i-p, k-i}(x)=0 .
$$

If $k>i, c_{p, i-p, k-i}(x)=c_{i-p, p, k-i}(x)=0$ for $1 \leq p \leq i-1$ by assumption (4.8). If $k$ $=i, \quad c_{p, i-p, 0}(x)=c_{p, 0, i-p}(x)=c_{0, p, i-p}(x)=c_{i-p, p, 0}(x)$ for $1 \leq p \leq i-1$ by the hypothesis of induction. Anyway, (4.10) holds for $i$. Then, $P(x, \xi)$ is $\widetilde{S}_{3}$-invariant with respect to $\xi$.

\section{Step 2. $P(x, \xi)$ is even for $B_{3}, D_{3}$ cases.}

We apply Proposition 4.1 for $\alpha=e_{i}+e_{j}$ and prove $P(x, \xi)$ is even with respect to $\xi$. For this, we prove that $c_{0, i, k-i}(x)=0$ if $i$ is odd by induction on $i$.

The coefficient of $\xi_{i}^{l-1} \xi_{h}^{k-l}$ in $\lim _{\xi_{j} \rightarrow-\xi_{i}}\left(\partial_{\xi_{i}}+\partial_{\xi_{j}}\right) P(x, \xi)=0$ is

$$
\begin{cases}\sum_{p=0}^{l}(-1)^{l-p}(2 p-l) c_{p, l-p, k-l}(x)=0 & (1 \leq l \leq k), \\ \sum_{p=0}^{l}(-1)^{l-p}(2 p-l) c_{k-l, p, l-p}(x)=0 & (1 \leq l \leq k), \\ \sum_{p=0}^{l}(-1)^{l-p}(2 p-l) c_{p, k-l, l-p}(x)=0 & (1 \leq l \leq k) .\end{cases}
$$

Equations (4.9) and (4.11) with $l=1$ imply $c_{0,1, k-1}(x)=0$. The first equality of (4.11) with $l=2 i+1$ is

$$
\begin{aligned}
& \sum_{p=0}^{2 i+1}(-1)^{2 i+1-p}(2 p-2 i-1) c_{p, 2 i+1-p, k-2 i-1}(x) \\
& =2 \sum_{p=0}^{i}(-1)^{p-1}(2 p-2 i-1) c_{p, 2 i+1-p, k-2 i-1}(x)=0 .
\end{aligned}
$$

Here, we used the $\Im_{3}$-invariance. If $k>2 i+1$, then $c_{p, 2 i+1-p, k-2 i-1}(x)=0$ for $1 \leq$ $p \leq i$ by assumption (4.8). If $k=2 i+1$, then $c_{p, 2 i+1-p, 0}(x)=c_{0, p, 2 i+1-p}(x)=$ $c_{0,2 i+1-p, p}(x)=0$ for $1 \leq p \leq i$ by the hypothesis of induction, since $p$ or $2 i+1-p$ is odd. Then $c_{0,2 i+1, k-2 i-1}(x)=0$. By $\Im_{3}$-invariance, step 2 is proved.

\section{Step 3. $n>3$ cases.}

For $\sigma \in \mathbb{S}_{n}$, let $\sigma p=\left(p_{\sigma(1)}, \cdots, p_{\sigma(n)}\right)$. Let $\sigma_{i j}$ be the interchange of $i$ and $j$. 
If $p_{i}$ or $p_{j}$ is 0 , then $c_{\sigma_{i j} p}(x)=c_{p}(x)$ by step 1 . If both $p_{i}$ and $p_{j}$ are not 0 , then there exists $h \neq i, j$ such that $p_{h}=0$ by assumption (4.8). Applying step 1 for $i$, $j, h$, we have $c_{\sigma_{i j} p}(x)=c_{p}(x)$. This implies that $P(x, \xi)$ is $\mathbb{S}_{n}$-invariant. Analogously, we can prove that $P(x, \xi)$ is even for $B_{n}$ and $D_{n}$ cases.

Remark 4.3. (1) If we remove the assumption (4.8), there exist polynomials of $\xi$ which satisfy the divisible condition in Proposition 4.2 but are not $W$-invariant.

For example,

$$
Q=\xi_{1}^{2} \xi_{2} \xi_{3}-\frac{1}{3} \xi_{1}^{3}\left(\xi_{2}+\xi_{3}\right)+\frac{1}{6} \xi_{1}^{4}
$$

satisfies $\lim _{\xi_{j \rightarrow \xi_{i}}}\left(\partial_{\xi_{i}}-\partial_{\xi_{j}}\right) Q=0$ for $1 \leq i<j \leq 3$ but is not $\widetilde{S}_{3}$-invariant.

But, in this periodic case, we can show the following statement by direct computation: "If $P$ is defined on a open subset of $C^{3}$, commutes with $H$, and ord $P \leq 4$, it is $\widetilde{S}_{3}$-invariant and is contained in the commutative ring of Ochiai-Oshima-Sekiguchi." Then the author expects that we can weaken the order assumptions.

(2) On the other hand, when the parameters of potential functions are special, it is known that there exist higher order differential operators which commute with $H$ but are not $W$-invariant ([VSC]).

At last, we come to explain the main theorem of this section. This is the uniqueness theorem of commutative rings with periodic potentials.

Theorem 4.4. Let $(W, \Sigma)$ be a pair of a classical Weyl group and the corresponding root system. Let $H=1 / 2 \sum_{i=1}^{n} \partial_{x_{i}}^{2}+\sum_{\alpha \in \Sigma+} u_{\alpha}(\langle\alpha, x\rangle)\left(u_{\alpha}(t)=\right.$ $\left.u_{w \alpha}(t)\right)$ be a Laplacian, where all $u_{\alpha}(t)$ 's are non-constant periodic functions in the classification of Theorem 2.1. Suppose that $P$ is a holomorphic differential operator which is defined on a connected open subset of the domain where $H$ is defined, and commutes with $H$. Assume that principal symbol $\sigma(P)$ of $P$ satisfies the condition (4.7). Then $P$ has analytic continuation to the whole domain where $H$ is defined and is contained in the commutative ring constructed by Ochiai, Oshima and Sekiguchi.

Proof. If $\sigma(P)$ satisfies (4.7), $\sigma(P)$ is constant with respect to $x$ and $W$-invariant with respect to $\xi$ by Corollary 3.2.(3) and Proposition 4.2. As a consequence of Ochiai, Oshima and Sekiguchi, we know that there exists an operator $P^{\prime}$ which commutes with $H$ and has the same principal symbol as $P$. The order of $P-P^{\prime}$ is lower than $P$, and $P-P^{\prime}$ commutes with $H$. Since $P$ satisfies the assumption (4.7), $P-P^{\prime}$ also. By the explicit formulae of higher order operators ([OOS, Theorem 2]), we know that $P^{\prime}$ is defined on the domain 
where $H$ is defined. Then this theorem is proved inductively.

\section{$\S 5 . \quad$ Rational Potential Cases}

In the rational potential case, the situation is a little more complicated than the periodic cases.

In the first half of this section, all the operators with order at most 2 are constucted, which commute with

$$
H=\frac{1}{2} \sum_{i=1}^{n} \partial_{x_{i}}^{2}+\sum_{1 \leq i<j \leq n}\left\{C_{1}\left(x_{i}-x_{j}\right)^{-2}+C_{2}\right\} .
$$

In the latter half of this section, using these operators, we investigate whether there exist commutative rings which satisfy (C1)-(C5) in $\$ 2$ but different from what Oshima and Sekiguchi constructed in [OS]. Note that their commutative ring is generated by

$$
\left\{\begin{array}{l}
\Delta_{1}=\sum_{i} \partial_{x_{i}} \\
H=\frac{1}{2} \sum_{i} \partial_{x_{i}}^{2}+\sum_{i<j} u\left(x_{i}-x_{j}\right), \\
\Delta_{3}=\sum_{i<j<k} \partial_{x_{i}} \partial_{x_{j}} \partial_{x_{k}}-\sum_{i}\left(\sum_{\substack{j<k \\
j, i \\
k \neq i}} u\left(x_{j}-x_{k}\right)\right) \partial_{x_{i}} \\
\cdots \cdots
\end{array}\right.
$$

where $u(t)=C_{1} t^{-2}+C_{2}$.

We use notation ir $\S \S 3,4$, and assume that $C_{1} \neq 0, n \geq 3$.

Suppose that $P=\sum_{k=0}^{m} P_{k}$ commutes with $H$. Then $\widetilde{P}_{m}$ satisfies (4.2). Since $\widetilde{Q}_{m-2}^{\prime}$ is a polynomial with respect to $x$ but $U_{\alpha}(t)$ has a pole at $t=0$, we have

$$
\lim _{\langle\alpha, x\rangle \rightarrow 0} \lim _{\langle\alpha, \xi\rangle \rightarrow 0}\left\langle\alpha, \partial_{x}\right\rangle \widetilde{P}_{m}=0 .
$$

Next, by (3.8), there exists $\widetilde{Q}_{m-2}^{\prime \prime} \in \mathscr{R}$ such that

$$
\lim _{\langle\alpha, \xi\rangle \rightarrow 0}\left\{\left\langle\alpha, \partial_{\xi}\right\rangle \widetilde{P}_{m} u_{\alpha}(\langle\alpha, x\rangle)+\frac{\left\langle\alpha, \partial_{x}\right\rangle \widetilde{P}_{m}}{\langle\alpha, \xi\rangle} U_{\alpha}(\langle\alpha, x\rangle)+\frac{\widetilde{Q}_{m-2}^{\prime \prime}}{\langle\alpha, \xi\rangle}\right\}=0 .
$$

Since $\lim _{\langle\alpha, \xi\rangle \rightarrow 0}\left\{\left\langle\alpha, \partial_{x}\right\rangle \widetilde{P}_{m} U_{\alpha}(\langle\alpha, x\rangle)+\widetilde{Q}_{m-2}^{\prime \prime}\right\}$ has no pole at $\langle\alpha, x\rangle=0$ by (5.2),

$$
\lim _{\langle\alpha, x\rangle \rightarrow 0} \lim _{\langle\alpha, \xi\rangle \rightarrow 0}\left\langle\alpha, \partial_{\xi}\right\rangle \widetilde{P}_{m}=0 .
$$

As we mentioned at the beginning of this section, we shall find all the operators $P$ with order at most 2 . We express $P$ as

$$
P=\sum_{i=1}^{n} a_{2}^{i} \partial_{x_{i}}^{2}+\sum_{1 \leq i<j \leq n} a_{11}^{i j} \partial_{x_{i}} \partial_{x_{j}}+\sum_{i=1}^{n} a_{1}^{i} \partial_{x_{i}}+a_{0} .
$$

Here, $a_{2}^{i}, a_{11}^{i j}, a_{1}^{i}$ and $a_{0}$ are holomorphic functions on $\left\{x \in \boldsymbol{C}^{n} ;|x|<r\right\} \cap\left(\boldsymbol{C}^{n}\right.$ 
$\left.-\bigcup_{1 \leq i<j \leq n}\left\{x \in C^{n} ; x_{i}=x_{j}\right\}\right)$

The coefficients of $\partial_{x}^{p}$ 's in $[H, P]=0$ are

$$
\begin{aligned}
\partial_{x_{i}}^{3}: & \partial_{x_{i}} a_{2}^{i}=0, \\
\partial_{x_{i}}^{2} \partial_{x_{j}}: & \partial_{x_{j}} a_{2}^{i}+\partial_{x_{i}} a_{11}^{i j}=0, \\
\partial_{x_{i}} \partial_{x_{j}} \partial_{x_{k}}: & \partial_{x_{k}} a_{11}^{i j}+\partial_{x_{j}} a_{11}^{i k}+\partial_{x_{i}} a_{11}^{j k}=0, \\
\partial_{x_{i}}^{2}: & \frac{1}{2} \sum_{p=1}^{n} \partial_{x_{p}}^{2} a_{2}^{i}+\partial_{x_{i}} a_{1}^{i}=0, \\
\partial_{x_{i}} \partial_{x_{j}}: & \frac{1}{2} \sum_{p=1}^{n} \partial_{x_{p}}^{2} a_{11}^{i j}+\partial_{x_{i}} a_{1}^{j}+\partial_{x_{j}} a_{1}^{i}=0, \\
\partial_{x_{i}}: & \frac{1}{2} \sum_{p=1}^{n} \partial_{x_{p}}^{2} a_{1}^{i}+\partial_{x_{i}} a_{0}=2 a_{2}^{i} \partial_{x_{i}} R(x)+\sum_{\substack{j=1 \\
j \neq i}}^{n} a_{11}^{i j} \partial_{x_{j}} R(x), \\
\text { constant }: & \frac{1}{2} \sum_{p=1}^{n} \partial_{x_{p}}^{2} a_{0} \\
& =\left(\sum_{i=1}^{n} a_{2}^{i} \partial_{x_{i}}^{2}+\sum_{1 \leq i<j \leq n} a_{11}^{i j} \partial_{x_{i}} \partial_{x_{j}}+\sum_{i=1}^{n} a_{1}^{i} \partial_{x_{i}}\right) R(x) .
\end{aligned}
$$

For $\alpha=e_{i}-e_{j},(5.4)$ is equivalent to

$$
\begin{aligned}
& \lim _{x_{j} \rightarrow x_{i}}\left(a_{2}^{i}-a_{2}^{j}\right)=0, \\
& \lim _{x_{j} \rightarrow x_{i}}\left(a_{11}^{i k}-a_{11}^{j k}\right)=0,
\end{aligned}
$$

for $i \neq j \neq k \neq i$. Notice that $a_{2}^{i}$ and $a_{11}^{i j}$ are polynomial functions of degree at most 2 by Corollary 3.2.(2).

Since $\partial_{x_{i}} a_{2}^{i}=\partial_{x_{j}} a_{2}^{j}=0$ from (5.6), $a_{2}^{i}=\lim _{x_{i} \rightarrow x_{j}} a_{2}^{i}=\lim _{x_{i} \rightarrow x_{j}} a_{2}^{j}=\sigma_{i j}\left(a_{2}^{j}\right)$ by (5.13). Here, $\sigma_{i j}$ is the interchange of $x_{i}$ and $x_{j}$. Then $a_{2}^{i}=\sigma_{i j}\left(a_{2}^{j}\right)=\sigma_{i j} \sigma_{j k}\left(a_{2}^{k}\right)$ $=\sigma_{i j} \sigma_{j k} \sigma_{k i}\left(a_{2}^{i}\right)=\sigma_{j k}\left(a_{2}^{i}\right)$, and this implies $a_{2}^{i}$ is a symmetric polynomial of $x_{1}, \cdots$, $\bar{x}_{i}, \cdots, x_{n}$. Then, for any $i, a_{2}^{i}$ is expressed as

$$
a_{2}^{i}=\mu_{1} \sum_{\substack{j=1 \\ j \neq i}}^{n} x_{j}^{2}+\mu_{2} \sum_{\substack{i \leq j<k \leq n \\ j, k \neq i}} x_{j} x_{k}+\mu_{3} \sum_{\substack{j=1 \\ j \neq i}}^{n} x_{j}+\mu_{4} .
$$

Here, $\mu_{p}$ 's do not depend on $i$ since $a_{2}^{i}=\sigma_{i j}\left(a_{2}^{j}\right)$.

Next, by (5.7), $\partial_{x_{i}} a_{11}^{i j}=-2 \mu_{1} x_{j}-\mu_{2} \sum_{\substack{k=1 \\ k \neq i, j}}^{n} x_{k}-\mu_{3}$. Define $\bar{a}_{11}^{i j}$ by

$$
\bar{a}_{11}^{i j}=a_{11}^{i j}+2 \mu_{1} x_{i} x_{j}+\mu_{2}\left(x_{i}+x_{j}\right) \sum_{\substack{k=1 \\ k \neq i, j}}^{n} x_{k}+\mu_{3}\left(x_{i}+x_{j}\right) .
$$

By (5.7) and (5.14), $\partial_{x_{i}} \bar{a}_{11}^{i j}=\partial_{x_{j}} \bar{a}_{11}^{i j}=0$, and $\lim _{x_{j} \rightarrow x_{i}}\left(\bar{a}_{11}^{i k}-\bar{a}_{11}^{j k}\right)=0$. By analogous argument as above, $\bar{a}_{11}^{i j}=\sigma_{i k}\left(\bar{a}_{11}^{j k}\right)$ and then $\bar{a}_{11}^{i j}$ is a symmetric polynomial of $x_{1}, \cdots, \widehat{x}_{i}, \cdots, \bar{x}_{j} \cdots, x_{n}$. Using (5.8), we have 


$$
\begin{aligned}
a_{11}^{i j}=-2 \mu_{1} x_{i} x_{j}-\mu_{2}\left(x_{i}+x_{j}\right) \sum_{\substack{k=1 \\
k \neq i, j}}^{n} x_{k} \\
-\mu_{3}\left(x_{i}+x_{j}\right)+\mu_{2} \sum_{\substack{k=1 \\
k \neq i, j}}^{n} x_{k}^{2}+\mu_{5} .
\end{aligned}
$$

Define $\bar{a}_{1}^{i}$ by

$$
a_{1}^{i}=\bar{a}_{1}^{i}-(n-1) \mu_{1} x_{i}-\frac{n-2}{2} \mu_{2} \sum_{\substack{j=1 \\ j \neq i}}^{n} x_{j} .
$$

Then by (5.9) and (5.10), $\bar{a}_{1}^{i}$ satisfies $\partial_{x_{i}} \bar{a}_{1}^{i}=\partial_{x_{j}} \bar{a}_{1}^{i}+\partial_{x_{i}} \bar{a}_{1}^{j}=0$. This implies that $\bar{a}_{1}^{i}$ is a polynomial function of degree at most 1 .

Finally, we determine $a_{0}$. From (5.11),

$$
\begin{aligned}
& \partial_{x_{i}} a_{0}= \sum_{\substack{j=1 \\
j \neq i}}^{n} \frac{-2 C_{1}\left(2 a_{2}^{i}-a_{11}^{i j}\right)}{\left(x_{i}-x_{j}\right)^{3}}+\sum_{\substack{1, j<k \leq n \\
j, k \neq i}} \frac{-2 C_{1}\left(a_{11}^{i j}-2 a_{11}^{i k}\right)}{\left(x_{j}-x_{k}\right)^{3}} \\
&=\sum_{\substack{j=1 \\
j \neq i}}^{n} \frac{-2 C_{1}}{\left(x_{i}-x_{j}\right)^{3}}\left[2 \mu_{1}\left(x_{i} x_{j}+\sum_{\substack{p=1 \\
p \neq i}}^{n} x_{p}^{2}\right)\right. \\
&+\mu_{2}\left\{2 \sum_{\substack{1 \leq p<q \leq n \\
p, q \neq i}} x_{p} x_{q}+\left(x_{i}+x_{j}\right) \sum_{\substack{p=1 \\
p \neq i, j}}^{n} x_{p}-\sum_{\substack{p=1 \\
p \neq i, j}}^{n} x_{p}^{2}\right\} \\
&\left.\quad+\mu_{3}\left(x_{i}+3 x_{j}+2 \sum_{\substack{p=1 \\
p \neq i, j}}^{n} x_{p}\right)+2 \mu_{4}-\mu_{5}\right] \\
&+\sum_{\substack{1 \leq j<k \leq n \\
j, k \neq i}} \frac{-2 C_{1}}{\left(x_{j}-x_{k}\right)^{2}}\left\{-2 \mu_{1} x_{i}+\mu_{2}\left(x_{i}-\sum_{\substack{p=1 \\
p \neq i}}^{n} x_{p}\right)-\mu_{3}\right\} .
\end{aligned}
$$

By this equation, we have

$$
\begin{aligned}
a_{0}=\sum_{1 \leq i<j \leq n} \frac{C_{1}}{\left(x_{i}-x_{j}\right)^{2}} & \left\{2 \mu_{1} \sum_{p=1}^{n} x_{p}^{2}\right. \\
& +\mu_{2}\left(2 \sum_{1 \leq p<q \leq n} x_{p} x_{q}-\sum_{p=1}^{n} x_{p}^{2}\right) \\
& \left.+2 \mu_{3} \sum_{p=1}^{n} x_{p}+2 \mu_{4}-\mu_{5}\right\}+\mu_{7} .
\end{aligned}
$$

(5.12) is equivalent to $-2 C_{1} \sum_{1 \leq i<j \leq n}\left(\bar{a}_{1}^{i}-\bar{a}_{1}^{j}\right)\left(x_{i}-x_{j}\right)^{-3}=0$. Since $\bar{a}_{1}^{i}$ is a polynomial function of degree at most 1 and satisfies $\partial_{x_{i}} \bar{a}_{1}^{i}=\partial_{x_{j}} \bar{a}_{1}^{i}+\partial_{x_{i}} \bar{a}_{1}^{j}=0$, $\bar{a}_{1}^{i}$ is a constant $\mu_{6}$, which does not depend on $i$.

Proposition 5.1. Let $P$ be a holomorphic differential operator of order at most 2 which is defined on $\left\{x \in C^{n} ;|x|<r\right\} \cap\left(C^{n}-\cup_{1 \leq i<j \leq n}\left\{x \in C^{n} ; x_{i}=\right.\right.$ $\left.\left.x_{j}\right\}\right)$ for some $r \in \boldsymbol{R}_{>0}$, and commutes with $H$. Then $P$ is $\subseteq_{n}$-invariant, has analytic continuation to $C^{n}-\bigcup_{1 \leq i<j \leq n}\left\{x \in C^{n} ; x_{i}=x_{j}\right\}$, and is a linear combi- 
nation of $1, \Delta_{1}=\sum_{i=1}^{n} \partial_{x_{i}}, \Delta_{1}^{2}, H, P_{1}, P_{2}$ and $P_{3}$, where

$$
\begin{aligned}
P_{1}:= & \sum_{1 \leq i<j \leq n}\left(x_{j} \partial_{x_{i}}-x_{i} \partial_{x_{j}}\right)^{2}+2 C_{1} \sum_{\substack{1 \leq i<j \leq n \\
1 \leq p \leq n}} x_{p}^{2}\left(x_{i}-x_{j}\right)^{-2}, \\
P_{2}:= & \sum_{1 \leq i \neq j \neq k \neq i \leq n}\left(x_{j} \partial_{x_{i}}-x_{i} \partial_{x_{j}}\right)\left(x_{k} \partial_{x_{i}}-x_{i} \partial_{x_{k}}\right) \\
& +2 C_{1} \sum_{1 \leq i<j \leq n}\left(2 \sum_{1 \leq p<q \leq n} x_{p} x_{q}-\sum_{p=1}^{n} x_{p}^{2}\right)\left(x_{i}-x_{j}\right)^{-2}, \\
P_{3}:= & \sum_{1 \leq i<j \leq n}\left(x_{j} \partial_{x_{i}}-x_{i} \partial_{x_{j}}\right)\left(\partial_{x_{i}}-\partial_{x_{j}}\right)+2 C_{1} \sum_{\substack{1 \leq i<j \leq n \\
1 \leq p \leq n}} x_{p}\left(x_{i}-x_{j}\right)^{-2} .
\end{aligned}
$$

For the construction of a commutative ring satisfying (C1)-(C5) in $\$ 2$ of $A_{n-1}$-type, we assume that $P$ commutes with $\Delta_{1}$, i.e.

$$
\Delta_{1} a_{2}^{i}=\Delta_{1} a_{11}^{i j}=\Delta_{1} a_{1}^{i}=\Delta_{1} a_{0}=0 .
$$

This implies $2 \mu_{1}+(n-2) \mu_{2}=\mu_{3}=0$. Then we have :

Proposition 5.2. All the differential operators which commute with $H$ and $\Delta_{1}$ are linear combinations of $1, H, \Delta_{1}, \Delta_{1}^{2}$ and

$$
\begin{gathered}
P=\sum_{1 \leq i<j<k \leq n}\left\{\left(x_{j}-x_{k}\right) \partial_{x_{i}}+\left(x_{k}-x_{i}\right) \partial_{x_{j}}+\left(x_{i}-x_{j}\right) \partial_{x_{k}}\right\}^{2} \\
\quad+2 C_{1} \sum_{\substack{1 \leq i<j \leq n \\
1 \leq p<q \leq n}}\left(x_{p}-x_{q}\right)^{2}\left(x_{i}-x_{j}\right)^{-2} .
\end{gathered}
$$

We shall prove that there is no commutative ring satisfying (C1)-(C5) in $\$ 2$, which contains $\Delta_{1}, H$ and $\Delta_{3}+\lambda_{1} P$, if $W=\Im_{n}, n>3$ and $\lambda_{1} \neq 0$.

Let

$$
\begin{aligned}
Q= & \sum_{1 \leq i<j<k<l \leq n} \partial_{x_{i}} \partial_{x_{j}} \partial_{x_{k}} \partial_{x_{l}}+\sum_{i=1}^{n} b_{3}^{i} \partial_{x_{i}}^{3}+\sum_{1 \leq i \neq j \leq n} b_{21}^{i j} \partial_{x_{i}}^{2} \partial_{x_{j}} \\
& +\sum_{1 \leq i<j<k \leq n} b_{111}^{i j k} \partial_{x_{i}} \partial_{x_{j}} \partial_{x_{k}}+\sum_{i=1}^{n} b_{2}^{i} \partial_{x_{i}}^{2}+\sum_{1 \leq i<j \leq n} b_{11}^{i j} \partial_{x_{i}} \partial_{x_{j}} \\
& +(\text { lower order terms })
\end{aligned}
$$

be a $\Im_{n}$-invariant differential operator which commutes with $\Delta_{1}, H$ and $\Delta_{3}$ $+\lambda_{1} P$. Note that $b_{3}^{i}, b_{21}^{i j}$ and $b_{111}^{i j k}$ are polynomials of $x$ with degree at most 3 by Corollary 3.2.(2).

The coefficients of $[H, Q]=0$ are

$$
\begin{gathered}
\partial_{x_{i}}^{4}: \partial_{x_{i}} b_{3}^{i}=0, \\
\partial_{x_{i}}^{3} \partial_{x_{j}}: \partial_{x_{j}} b_{3}^{i}+\partial_{x_{i}} b_{21}^{i j}=0,
\end{gathered}
$$




$$
\begin{aligned}
& \partial_{x_{i}}^{2} \partial_{x_{j}}^{2}: \partial_{x_{j}} b_{21}^{i j}+\partial_{x_{i}} b_{21}^{j i}=0 \text {, } \\
& \partial_{x_{i}}^{2} \partial_{x_{j}} \partial_{x_{k}}: \partial_{x_{k}} b_{21}^{i j}+\partial_{x_{j}} b_{21}^{i k}+\partial_{x_{i}} b_{111}^{i j k}=0 \text {, } \\
& \text { (5.19) } \quad \partial_{x_{i}} \partial_{x_{j}} \partial_{x_{k}} \partial_{x_{l}}: \partial_{x_{l}} b_{111}^{i j k}+\partial_{x_{k}} b_{111}^{i j l}+\partial_{x_{j}} b_{111}^{i k l}+\partial_{x_{i}} b_{111}^{j k l}=0 \text {, } \\
& \partial_{x_{i}}^{3}: \frac{1}{2} \sum_{p=1}^{n} \partial_{x_{p}}^{2} b_{3}^{i}+\partial_{x_{i}} b_{2}^{i}=0, \\
& \partial_{x_{i}}^{2} \partial_{x_{j}}: \frac{1}{2} \sum_{p=1}^{n} \partial_{x_{p}}^{2} b_{21}^{i j}+\partial_{x_{j}} b_{2}^{i}+\partial_{x_{i}} b_{11}^{i j}=0, \\
& \partial_{x_{i}} \partial_{x_{j}} \partial_{x_{k}}: \frac{1}{2} \sum_{p=1}^{n} \partial_{x_{p}}^{2} b_{111}^{i j k}+\partial_{x_{k}} b_{11}^{i j}+\partial_{x_{j}} b_{11}^{i k}+\partial_{x_{i}} b_{11}^{j k} \\
& =2 C_{1} \sum_{p=i, j, k} \sum_{\substack{q=1 \\
q \neq i, j, k}}^{n}\left(x_{p}-x_{q}\right)^{-3},
\end{aligned}
$$

respectively. The coefficients of $\left[\Delta_{3}+\lambda_{1} P, Q\right]=0$ are

$$
\partial_{x_{i}}^{4} \partial_{x_{j}}: \sum_{p \neq i, j} \partial_{x_{p}} b_{3}^{i}=0,
$$

$$
\begin{gathered}
\partial_{x_{i}}^{3} \partial_{x_{j}} \partial_{x_{k}}: \sum_{p \neq j, k} \partial_{x_{p}} b_{3}^{i}+\sum_{p \neq i, k} \partial_{x_{p}} b_{21}^{i j}+\sum_{p \neq i, j} \partial_{x_{p}} b_{21}^{i k} \\
=\sum_{p \neq i, j, k} \partial_{x_{p}} a_{2}^{i}, \\
\partial_{x_{i}}^{3} \partial_{x_{j}}: \sum_{\substack{p<q \\
p, q \neq j}} \partial_{x_{p}} \partial_{x_{q}} b_{3}^{i}+\sum_{\substack{p<q \\
p, q \neq i}} \partial_{x_{p}} \partial_{x_{q}} b_{21}^{i j}+\sum_{p \neq i, j} \partial_{x_{p}} b_{2}^{i}+2 a_{2}^{j} \partial_{x_{j}} b_{3}^{i} \\
\quad+\sum_{p \neq j} a_{11}^{j p} \partial_{x_{p}} b_{3}^{i}+2 a_{2}^{i} \partial_{x_{i}} b_{21}^{i j}+\sum_{p \neq i} a_{11}^{i p} \partial_{x_{p}} b_{21}^{i j} \\
=3 b_{3}^{i} \partial_{x_{i}} a_{11}^{i j}+\sum_{p \neq i} b_{21}^{i p} \partial_{x_{p}} a_{11}^{i j}+2 b_{21}^{i j} \partial_{x_{i}} a_{2}^{i} \\
+2 b_{21}^{j i} \partial_{x_{j}} a_{2}^{i}+\sum_{p \neq i, j} b_{111}^{i j p} \partial_{x_{p}} a_{2}^{i},
\end{gathered}
$$

respectively. Since $\left[\Delta_{1}, Q\right]=0, \Delta_{1} b_{3}^{i}=\Delta_{1} b_{21}^{i j}=\Delta_{1} b_{111}^{i j h}=0$. By (5.15), (5.23) and $\Delta_{1} b_{3}^{i}=0$,

$$
b_{3}^{i}=\lambda_{2} \quad \text { (constant). }
$$

Moreover, by (5.16), (5.18) and (5.19),

$$
\begin{aligned}
\partial_{x_{i}} b_{21}^{i j} & =0, \\
\partial_{x_{i}}^{2} b_{111}^{i j k} & =\partial_{x_{i}}^{3} b_{111}^{i j k}=0 .
\end{aligned}
$$

Proposition 5.2, (5.18), (5.24), (5.27) and $\Delta_{1} b_{21}^{i j}=0$ imply

$$
\begin{aligned}
\partial_{x_{i}} b_{111}^{i j k} & =-\partial_{x_{k}} b_{21}^{i j}-\partial_{x_{j}} b_{21}^{i k}=-\left(\partial_{x_{j}}+\partial_{x_{k}}\right) a_{2}^{i} \\
& =-2 \lambda_{p} \sum_{p \neq i, j, k}\left(x_{j}+x_{k}-2 x_{p}\right) .
\end{aligned}
$$

Then $b_{111}^{i j k}$ can be written as 


$$
\begin{aligned}
b_{111}^{i j k}=- & 2(n-3) \lambda_{1}\left(x_{i} x_{j}+x_{j} x_{k}+x_{k} x_{i}\right) \\
& +4 \lambda_{1}\left(x_{i}+x_{j}+x_{k}\right) \sum_{p \neq i, j, k} x_{p}+\bar{b}_{111}^{i j k},
\end{aligned}
$$

where $\bar{b}_{111}^{i j k}$ is a symmetric polynomial of $n-3$ variables $x_{p}(1 \leq p \leq n, p \neq i, j$, $k$ ) with degree at most 3. By $\Delta_{1} b_{111}^{i j k}=0,(5.19)$ and (5.27),

$$
\bar{b}_{111}^{i j k}=-6 \lambda_{1} \sum_{\substack{p=1 \\ p \neq i, j, k}}^{n} x_{p}^{2}+\lambda_{3}
$$

Then

$$
\begin{aligned}
b_{111}^{i j k}=- & 2(n-3) \lambda_{1}\left(x_{i} x_{j}+x_{j} x_{k}+x_{k} x_{i}\right) \\
& +4 \lambda_{1}\left(x_{i}+x_{j}+x_{k}\right) \sum_{p \neq i, j, k} x_{p}-6 \lambda_{1} \sum_{p \neq i, j, k}^{n} x_{p}^{2}+\lambda_{3} .
\end{aligned}
$$

Next, we determine $b_{21}^{i j}$. By $\partial_{x_{i}} b_{21}^{i j}=\partial_{x_{j}}^{2} b_{21}^{i j}=0$, (5.17) and symmetry, $\partial_{x_{j}} b_{21}^{i j}=$ $\partial_{x_{i}} b_{21}^{j i}=0$.

From $\partial_{x_{k}} b_{21}^{i j}+\partial_{x_{j}} b_{21}^{i k}=2 \lambda_{1} \sum_{p \neq i, j, k}\left(x_{j}+x_{k}-2 x_{p}\right)$, we have $\partial_{x_{k}}^{2} b_{21}^{i j}=2(n-3) \lambda_{1}$ and $\partial_{x_{k}} \partial_{x_{l}} b_{21}^{i j}=-2 \lambda_{1}$, since $\partial_{x_{k}} \partial_{x_{l}} b_{21}^{i j}=-\partial_{x_{j}} \partial_{x_{l}} b_{21}^{i k}-4 \lambda_{1}=\partial_{x_{j}} \partial_{x_{k}} b_{21}^{i l}=-\partial_{x_{k}} \partial_{x_{l}} b_{21}^{i j}$ $-4 \lambda_{1}$. Then

$$
b_{21}^{i j}=\lambda_{1} \sum_{\substack{p<<q \\ p, q \neq i, j}}\left(x_{p}-x_{q}\right)^{2}+\lambda_{4} \sum_{p \neq i, j} x_{p}+\lambda_{5}
$$

$\Delta_{1} b_{21}^{i j}=0$ implies $\lambda_{4}=0$, and we have

$$
b_{21}^{i j}=\lambda_{1} \sum_{\substack{p<q \\ p, q \neq i, j}}\left(x_{p}-x_{q}\right)^{2}+\lambda_{5}
$$

Define $\bar{b}_{11}^{i j}$ by

$$
\bar{b}_{11}^{i j}=\sum_{\substack{p<q \\ p, q \neq i, j}}\left\{C_{1}\left(x_{p}-x_{q}\right)^{-2}+C_{2}\right\}+(n-3) \lambda_{1} \sum_{p \neq i, j}\left(x_{i}+x_{j}-2 x_{p}\right)+b_{11}^{i j} .
$$

Then by (5.20), (5.21), (5.22), (5.26), (5.28) and (5.29),

$$
\begin{aligned}
& \partial_{x_{i}} b_{2}^{i}=0, \\
& \partial_{x_{j}} b_{2}^{i}+\partial_{x_{i}} \bar{b}_{11}^{i j}=0, \\
& \partial_{x_{k}} \bar{b}_{11}^{i j}+\partial_{x_{j}} \bar{b}_{11}^{i k}+\partial_{x_{i}} \bar{b}_{11}^{j k}=0 .
\end{aligned}
$$

This is equivalent to

$$
\left\{\frac{1}{2} \sum_{i} \xi_{i}^{2}, \sum_{i} b_{2}^{i} \xi_{i}^{2}+\sum_{i<j} \bar{b}_{11}^{i j} \xi_{i} \xi_{j}\right\}=0
$$

then $b_{2}^{i}$ and $\bar{b}_{11}^{i j}$ are polynomials of $x$ with degree at most 2 .

Since $\Delta_{1} b_{2}^{i}=\Delta_{1} \bar{b}_{11}^{i j}=0$,

$$
b_{2}^{i}=\lambda_{6} \sum_{\substack{p<q \\ p, q \neq i}}\left(x_{p}-x_{q}\right)^{2}+\lambda_{7}
$$




$$
\bar{b}_{11}^{i j}=-2 \lambda_{6} \sum_{p \neq i, j}\left(x_{p}-x_{i}\right)\left(x_{p}-x_{j}\right)+\lambda_{8}
$$

Differentiating both hand side of $(5.25)$ by $x_{j}$ three times, and using Proposition 5.2, (5.26), (5.28), (5.29) and (5.30), we have

$$
\begin{aligned}
0 & =\partial_{x_{j}}^{3}\left(\sum_{p \neq i} b_{21}^{i p} \partial_{x_{p}} a_{11}^{i j}\right)=3 \sum_{p \neq i} \partial_{x_{j}}^{2} b_{21}^{i p} \partial_{x_{j}} \partial_{x_{p}} a_{11}^{i j} \\
& =3 \sum_{p \neq i, j} 2 \lambda_{1}(n-3) \cdot 2 \lambda_{1}=12(n-2)(n-3) \lambda_{1}^{2} .
\end{aligned}
$$

Then $\lambda_{1}=0$. By Theorem 3.2 of [OS], the commutative ring which contains $\Delta_{1}$, $H$ and $\Delta_{3}+\lambda_{1} P$ and satisfies (C1)-(C5) in $\S 2$ is unique. Then we have proved:

Theorem 5.3. Suppose that $W=\Im_{n}$ and $u(t)=C_{1} t^{-2}+C_{2}$.

(1) If $n>3$, then there exists no ring satisfying (C1)-(C5) in \$2 other than what Oshima and Sekiguchi constructed in [OS].

(2) If $n=3$, then $\Delta_{3}=\partial_{x_{1}} \partial_{x_{2}} \partial_{x_{3}}-C_{1} \sum_{\substack{1 \leq i \leq 3 \\ 1 \leq j<k \leq 3, j, k \neq \imath}}\left(x_{j}-x_{k}\right)^{-2} \partial_{x_{i}}, \Delta_{1}$ and $H$ generate a commutative ring. This is proved in [OS]. Define

$$
\begin{gathered}
P=\left\{\left(x_{2}-x_{3}\right) \partial_{x_{1}}+\left(x_{3}-x_{1}\right) \partial_{x_{2}}+\left(x_{1}-x_{2}\right) \partial_{x_{3}}\right\}^{2} \\
+2 C_{1} \sum_{\substack{1 \leq i<j \leq 3 \\
1 \leq p<q \leq 3}}\left(x_{p}-x_{q}\right)^{2}\left(x_{i}-x_{j}\right)^{-2} .
\end{gathered}
$$

Then, $\Delta_{1}, H$ and $\Delta_{3}+\lambda_{1} P$ also generate a commutative ring satisfying (C1)-(C5) in $\$ 2$ for any $\lambda_{1}$.

Remark 5.4. For $B_{n}$ and $D_{n}$ cases, it is not difficult to obtain the corresponding results to Proposition 5.1 and Theorem 5.3. Let $P$ be a holomorphic differential operator of order at most 2 which commutes with $H=1 / 2 \sum_{i=1}^{n} \partial_{x_{i}}^{2}$ $+\sum_{1 \leq i<j \leq n}\left\{u\left(x_{i}+x_{j}\right)+u\left(x_{i}-x_{j}\right)\right\}+\sum_{i=1}^{n} v\left(x_{i}\right)$, where $u(t)=C_{0}+C_{1} t^{-2}+C_{2} t^{2}$ and $v(t)=B_{0}+B_{1} t^{-2}+B_{3} t^{2}$ (see Theorem 2.1 (2.3)). If $C_{1} \neq 0$, then $P$ is a linear combination of $1, H$ and

$$
\begin{aligned}
P_{1}= & \sum_{1 \leq i<j \leq n}\left(x_{j} \partial_{x_{i}}-x_{i} \partial_{x_{j}}\right)^{2} \\
& +2\left(\sum_{p=1}^{n} x_{p}^{2}\right)\left\{C_{1} \sum_{1 \leq i<j \leq n}\left(\left(x_{i}+x_{j}\right)^{-2}+\left(x_{i}-x_{j}\right)^{-2}\right)+B_{1} \sum_{i=1}^{n} x_{i}^{-2}\right\} .
\end{aligned}
$$

Moreover, all the commutative rings satisfying (C1)-(C5) in $\$ 2$ are constructed in $[\mathrm{OO}]$ and $[\mathrm{OS}]$.

\section{References}

[OO] Ochiai, H. and Oshima, T., Commuting differential operators of type $B_{2}$, Preprint, 1994, 
UTMS 94-65, Dept. of Mathematical Science, University of Tokyo.

[OOS] Ochiai, H., Oshima, T. and Sekiguchi, H., Commuting families of symmetric differential operators, Proc. Japan Acad., 70 A (1994), 62-66.

[OP1] Olshanetsky, M.A. and Perelomov, A.M., Quantum system connected with root systems and the radial parts of Laplace operators, Funct. Anal. Appl., 12 (1978), 60-68.

[OP2] _ Quantum integrable systems related to Lie algebras, Phys. Rep., 94 (1983), 313-404.

[OS] Oshima, T. and Sekiguchi, H., Commuting families of differential operators invariant under the action of a Weyl group, J. Math. Sci. Univ. Tokyo, 2 (1995), 1-75.

[T] Taniguchi, K., On uniqueness of commuting families of differential operators invariant under the action of $\mathfrak{S}_{n}$, Master Theses, University of Tokyo (1994).

[VSC] Veselov, A.P., Styrkas, K.L. and Chalykh, O.A., Algebraic integrability for the Schrödinger equation and finite reflection groups, Theoret. and Math. Phys., 94 (1993), 182-197.

[WW] Whittaker, E.T. and Watson, G.N., A Course of Modern Analysis, Fourth Edition, Cambridge University Press, 1927. 